\title{
Association between Salivary Fructosamine, Plasma Glycated Hemoglobin, and Plasma Glucose Levels among Type II Diabetes Mellitus and Nondiabetic Individuals-A Cross-sectional Study
}

\author{
Sadhana Kandavel ${ }^{1} \quad$ P. D. Madan Kumar ${ }^{2}$ \\ ${ }^{1}$ Department of Public Health Dentistry, Sree Balaji Dental College \\ and Hospital, Chennai, Tamil Nadu, India \\ ${ }^{2}$ Department of Public Health Dentistry, Ragas Dental College and \\ Hospital, Chennai, Tamil Nadu, India
}

\begin{abstract}
Address for correspondence Sadhana Kandavel, MDS, Department of Public Health Dentistry, Sree Balaji Dental College and Hospital, Chennai, Tamil Nadu 600100, India (e-mail: sadhanakandavel@gmail.com).
\end{abstract}

Eur J Dent 2019;13:310-317

\begin{abstract}
Keywords

- noninvasive

- saliva

- fructosamine

- diabetes mellitus

- glycated hemoglobin

Objective The study was aimed to determine the association between salivary fructosamine and plasma glycated hemoglobin, plasma fasting and postprandial glucose levels among patients suffering from type II diabetes mellitus and nondiabetic healthy individuals.

Materials and Methods The cross-sectional study was conducted in a hospital setting. A total of 100 participants were included, with 50 participants in each groupgroup I (patients with type II diabetes mellitus) and group II (nondiabetic healthy individuals). Blood and saliva samples were collected to estimate the levels of the blood and salivary parameters. Depending upon the normality, appropriate tests were used$t$-test and Mann-Whitney $U$ test were used for continuous data. Pearson chi-square test and Fisher's exact test were used for discrete data. To test for any positive association between salivary and blood parameters, simple linear regression was carried out.

Results The study results showed that group I had significantly higher levels of salivary fructosamine when compared to group II $(p<0.001)$. In groups I and II a significant positive association was observed between salivary fructosamine and plasma glycated hemoglobin (group I, r-value 0.893; group II, r-value 0.307 ).

Conclusion The overall study results showed that salivary fructosamine levels were significantly higher in patients with diabetes when compared to nondiabetic individuals. Also, positive correlation was observed between salivary fructosamine, plasma fasting, plasma postprandial, and plasma glycated hemoglobin among both the groups.
\end{abstract}

\section{Introduction}

Diabetes is the most common endocrine disorder across the world. According to the International Diabetes Federation (IDF), in the year 2014 an estimated 387 million people suffered from diabetes. There has been an alarming rise in the prevalence of all types of diabetes, and it has been projected that the number will increase by $55 \%$ by the year $2035 .{ }^{1}$
India has the world's largest population living with diabetes after China. ${ }^{2}$ The IDF reported that in the year 2014 about 66.8 million Indians suffered from diabetes and it may rise to 109 million by the year $2025 .{ }^{1}$

Diabetes has an asymptomatic stage that may be present for up to 7 years before it is diagnosed. ${ }^{3}$ Type-II diabetes mellitus frequently remains undetected for a long time, about half of the patients with diabetes present with one or more 
irreversible complications at the time of diagnosis. ${ }^{4-6}$ Hence early detection and periodic monitoring of blood glucose improve microvascular outcomes, and help both patients and their physicians assess the responses to therapy. ${ }^{1}$

In recent times the performance of the diagnostic test for diabetes has been reviewed extensively. Although the tests recommended by the American Diabetes Association (ADA) are widely used, each of them has their pros and cons, hence screening programs include a combination of these tests. ${ }^{6,7}$

All the conventional diagnostic tools apart from requiring a venous puncture, which is a major disadvantage, have other limitations. The limitations of fasting plasma glucose (FPG) testing are overnight fast and a $12 \%$ to $15 \%$ day-to-day variance in $\mathrm{FPG}^{3,5}$ The oral glucose tolerance test is considered a first-line diagnostic test; however, it has limitations such as poor patient compliance and reproducibility. ${ }^{8,9}$

Maillard reaction is the process by which carbonyl group of reducing sugars such as glucose react nonenzymatically with amino groups in proteins to form aldimines or early glycation products (Schiff's base). These early glycation products consequently undergo Amadori rearrangement and form intermediate glycation products which include plasma glycated hemoglobin (HbA1C) and plasma fructosamine. ${ }^{10}$

The advanced glycated end products (AGE) are formed by classic rearrangement of the intermediate glycation products. Pentosidine and pyralline are a few AGE products. ${ }^{10}$ Unlike Amadori products, AGE products affect long-living proteins. Thus, advanced glycation reflects glycemic control over a long period. However, at present there is no universally accepted method to detect AGE and no internationally recognized standard unit of measurement, making the comparison of results between different laboratories difficult. Hence plasma HbA1C and plasma fructosamine values are widely used to estimate the glycemic control throughout 1 to 8 weeks. ${ }^{10,11}$

Plasma glycated hemoglobin (HbA1C) is formed by the glycation of valine and lysine residues of hemoglobin. Similarly, plasma fructosamine is formed by the glycation of plasma protein albumin. The half-life of hemoglobin is 60 days, and that of albumin is 14 to 20 days. Thus, plasma HbA1C levels indicate the glycemic control for 6 to 8 weeks, and plasma fructosamine levels indicate the mean blood glucose concentration over 2 to 3 weeks. ${ }^{11}$

Plasma HbA1C measurement has been recently included by the ADA as a diagnostic and screening tool for diabetes. The chief advantages of using HbA1C measurement is the ease of testing because it does not require fasting and acute perturbations do not affect HbA1C levels; further microangiopathic complications strongly associate with $\mathrm{HbA1C}$ levels. ${ }^{12}$ The limitations of HbA1C testing include variation among different races. Also conditions such as anemia and some medications may influence the results.

Currently, plasma fructosamine levels are used to monitor the glycemic control throughout 1 to 3 weeks, as it is a more sensitive marker than plasma HbA1C. ${ }^{11}$ Plasma fructosamine is unaffected by disorders of red blood cells and medications ${ }^{13}$ The limitation of plasma fructosamine test is that the test results must be interpreted with caution in case of protein-losing disorders such as nephropathy and liver disorder and it requires venous puncture. ${ }^{11,13}$

Saliva, a valuable biofluid, contains abundant biomarkers, produced by the major and minor salivary glands, gingival crevicular fluid (GCF), mucosal transudations, serum and blood sheds from oral wounds, desquamated epithelial cells, acquired pellicles, bacterial products, viruses and fungi, other cellular components, and food debris. ${ }^{14}$ Saliva is often referred to as plasma ultrafiltrate, as about 1,000 different proteins and 19,000 unique peptide sequences have been detected in saliva. ${ }^{15}$ The mechanism by which the biomarkers enter the saliva includes passive diffusion, active transport, or extracellular ultrafiltration. As it provides a good reflection of the physiological functions of the body, human saliva is known as the "mirror of the body." 14,15

In recent times, substantial body of evidence, describes the use of saliva as a liquid biopsy, for the diagnosis of oral diseases like dental caries, gingivitis, periodontitis (chronic) aggressive), Bechet disease, oral squamous cell carcinoma, cleft palate and lips, salivary gland diseases, oral leukoplakia, chronic graft-versus-host disease (cGVHD), and systematic diseases such as breast cancer, diabetes, human immune deficiency virus (AIDS). ${ }^{15}$ Apart from diagnosis of diseases, saliva is also used to assess drug levels, toxicity, infectious, immunological, and hormonal levels, and in forensic investigations. The proteomic or genomic macromolecules present in saliva makes it a predictive, diagnostic, and prognostic tool for carcinomatous, inflammatory, and genetic disorders. ${ }^{14-16}$

Studies have reported that the glycated salivary proteins correlate with plasma glucose levels and plasma glycated proteins. Thus salivary fructosamine levels have been reported to correlate with plasma glucose and plasma HbA1C levels. ${ }^{17,18}$

Salivary fructosamine can be used like plasma HbA1C levels to monitor the glycemic control over a period of 1 to 3 weeks. Since the collection of saliva is simple and painless, it can be used as a noninvasive adjuvant tool for monitoring the glycemic control in diabetic patients, thus reducing the necessity of repeated venous punctures to draw blood. ${ }^{19}$

The five different types of oral fluid that can be utilized in the field of salivary diagnostics are (1) unstimulated whole saliva (UWS), (2) stimulated whole saliva (SWS), (3) saliva from parotid gland, (4) saliva from submandibular/ sublingual gland, and (5) saliva from minor glands. ${ }^{20}$ Each of them, in turn, have a specific method of collection and type of collection device. Salivette device (Sarstedt, Numbrecht, Germany), Quantisal (Immunalysis, Pomona, California), and SCS (Greiner-BioOne, Kremsmunster, Austria) are the most commonly used collection devices. ${ }^{16,20} \mathrm{Groschl}$ et al have suggested that immediate processing of the sample is essential to avoid errors in the study due to sample instability issues, and added to this the adsorption behavior of saliva on various devices could negatively affect the sample integrity. ${ }^{21}$ Various studies have observed a significant difference in the analyte levels, depending on the collection method used. This has led to the invention of novel devices such as SuperSAL and VersiSAL technologies (Oasis Diagnostics Corporation, Vancouver, Washington). 
These devices provide a standardized specimen, resulting in a more consistent sample uniformity. This is made possible by the use an inert absorbent material, as the collection media, that effectively releases the analyte from the pad, in contrast to the cotton bases that cause interference with the test results. ${ }^{16,20}$

The various methods used to analyze biomarkers present in saliva are high-performance liquid chromatography (HPLC), enzyme-linked immunosorbent assay (ELISA), radioimmunoassay, two-dimensional electrophoresis (2DE), mass spectrometry (MS), 2DE and reverse-phase liquid chromatography (LC), LC-tandem MS, matrix-assisted laser desorption/ ionization time-off light mass spectrometry (MALDI-TOF MS), and 2DE followed by MALDI-TOF MS. The method used for analysis depends on the biomarker that is to be analyzed in saliva. ${ }^{22}$ At present, novel discoveries such as the lab on a chip system and other biosensor systems have opened new vistas to research in the field of personalized medicine and dentistry. ${ }^{22,23}$

A substantial body of evidence suggests that Asian Indians are genetically more susceptible to type II diabetes mellitus, mainly due to the Pro 12 Ala polymorphism which is not protective against diabetes or insulin resistance among Asian Indians. Coupled with the above factors, lack of physical activity and high fat sugar-rich diet has escalated the prevalence of type II diabetes to alarming levels in India. ${ }^{24,25}$

To the best of our knowledge, there are only a few studies conducted in India on the levels of salivary fructosamine amongst diabetes mellitus patients; also, by extensive literature review we could identify numerous research papers on the role of salivary biomarkers in cancer diagnosis. However, there is a huge lacuna in the volume of research on the potential use of saliva in the diagnosis and posttreatment monitoring of diabetes mellitus. It has been projected that with the growing population, India will soon become the diabetic capital of the world; hence we found a pressing need to conduct this study. ${ }^{1,2}$

The etiopathology of the two types of diabetes mellitus are different and type II diabetes mellitus being the most common form among the two, the present study was contemplated to determine the association between salivary fructosamine and plasma glycated hemoglobin, plasma fasting and postprandial glucose levels among type-II diabetes mellitus patients and nondiabetic individuals in Chennai.

\section{Materials and Methods}

This cross-sectional study was designed to determine the association between salivary fructosamine and plasma glycated hemoglobin, plasma fasting, and postprandial glucose levels among type II diabetes mellitus patients. The study participants were divided into two groups: group I (type II diabetes mellitus patients) and group II (nondiabetic individuals). The study was conducted for a period of 5 months.

Ethical clearance was obtained from the institution review board. Permission to collect blood and saliva was obtained from the hospital superintendent.
The inclusion and exclusion criteria of the study are as follows.

\section{Inclusion Criteria}

1. Patients with type II diabetes mellitus having plasma fasting glucose levels greater than or equal to $126 \mathrm{mg} / \mathrm{dL}$ were included in group I (ADA-standards of medical care). ${ }^{1}$

2. Nondiabetic healthy individuals (with no other systemic disease) having plasma fasting glucose levels less than $126 \mathrm{mg} / \mathrm{dL}$ were included in group II (ADA-Standards of medical care). ${ }^{1}$

3. Patients who were willing to participate in the study.

\section{Exclusion Criteria}

1. Type II diabetes mellitus patients with diabetes-associated complications such as protein-losing nephropathy and liver failure (confirmed by their hospital reports).

2. Patients with hemolytic anemia or hemoglobinopathy.

3. Patients who underwent a recent blood transfusion.

The sample size was calculated using the $G$ power statistical software The Pearson's correlation coefficient $(r=0.675)$, from a study conducted by Nakamoto et al was considered. ${ }^{19}$ On considering alpha error as 0.05 and power as $90 \%$, a sample size of 42 for each group was obtained. Hence we included 50 individuals in each group, with a total of 100 participants.

\section{Recruitment of Patients with Type II Diabetes Mellitus (Group I)}

Patients with type II diabetes who visited the Department of Diabetology at Aringar Anna Government Hospital Chennai were approached for the study.

Recruitment of Nondiabetic Healthy Individuals (Group II) Individuals who enrolled in the routine master health checkup conducted once a month by the general outpatient department (OPD)were approached for the study.

Informed consent (bilingual) was obtained from participants who met the inclusion criteria and were willing to participate in the study.

A prevalidated questionnaire (validated by Pan American Health Organization and World Health Organization) was administered to collect details about sociodemographic factors, family history of noncommunicable disease, tobacco usage, and alcohol consumption. (The demographic details relevant to the present study and questions related to diabetes mellitus have been included in Results section.)

\section{Collection of Saliva and Blood}

Blood and saliva samples were obtained only as a part of their routine periodic checkup (group I) and master health checkup (group II).

Blood samples were collected after an overnight fast. Blood was drawn from the antecubital vein, and it was collected in vacutainers by a trained phlebotomist. The blood samples were analyzed at the hospital laboratory. 
After the participants rinsed their mouth with water six times, $3 \mathrm{~mL}$ of unstimulated saliva samples were collected in a sterile container. ${ }^{19}$ The saliva thus obtained were transferred to a sample collection box containing gel packs and transported to the laboratory, to estimate the salivary fructosamine levels. The samples were stored under two to $8^{\circ} \mathrm{C}$ at the laboratory before carrying out the laboratory procedures.

\section{Laboratory Procedure}

The automated laboratory procedure was carried out by trained laboratory technicians, using the following types of equipment.

- Hexokinase-mediated reaction Roche/Hitachi modular P chemistry analyzer

- Autoanalyzer (fasting and postprandial plasma glucose levels)

- Tosoh A1C 2.2 plus glycohemoglobin analyzer

- HPLC (high-perfusion liquid chromatography) analyzer(plasma glycated hemoglobin level)

- NBT kinetic-Spectrophotometer, measuring at $520 \mathrm{~nm}$ (salivary fructosamine)

Statistical analysis was performed using SPSS version 20. (IBM Corp. Released 2013. IBM SPSS Statistics for Windows, Version 22.0. Armonk, NY: IBM Corp.) Shapiro-Wilk Normality test was run. Depending on the nature of the data, appropriate parametric and nonparametric tests were chosen, a $p$ value of 0.05 was considered to be significant. Simple linear regression was carried out to determine the association between salivary fructosamine and plasma glycated hemoglobin, plasma fasting, and postprandial glucose levels.

\section{Results}

The present study was conducted to determine the association between salivary fructosamine and plasma glycated hemoglobin, plasma fasting, and postprandial glucose levels among patients with type II diabetes mellitus and nondiabetic individuals.

- Table 1 shows the distribution based on the demographic details of the study population. Among the demographic information that was collected, there were no significant differences in age, sex, race, and tobacco and alcohol usage between the two groups. The family history of type II Diabetes mellitus was found to be significantly higher among individuals in group I when compared to group II.

- Table 2 shows the distribution based on the mean BMI (body mass index), plasma fasting, and postprandial glucose, plasma glycated hemoglobin, and salivary fructosamine levels of the study population. A significant difference in BMI, plasma fasting, and postprandial glucose, plasma glycated hemoglobin, and salivary fructosamine levels were observed among the two groups. In group I female patients had significantly higher levels of plasma fasting and postprandial glucose, plasma glycated hemoglobin, and salivary fructosamine when compared to male participants.
- Table 3 shows the correlation between salivary fructosamine, plasma fasting, and postprandial glucose, and plasma glycated hemoglobin levels in the two groups. In group I individuals high correlation was observed between salivary fructosamine, plasma fasting, postprandial glucose levels, and plasma glycated hemoglobin. In group II high correlation was observed between salivary fructosamine and plasma fasting glucose levels. A moderate correlation was observed between salivary fructosamine and plasma postprandial glucose and plasma glycated hemoglobin levels. -Fig. 1 and -Fig. 2 shows the correlation between salivary fructosamine and plasma glycated hemoglobin levels in group I and II individuals, respectively.

\section{Discussion}

To improve treatment outcomes and financial burden, there is the need for early diagnosis of diabetes, by periodic monitoring of glycemic control. ${ }^{26}$

A few studies have been conducted to estimate the levels of salivary glucose, and to determine the association between salivary glucose and blood glucose levels. However, due to the presence of bacteria and enzymes in the oral cavity, salivary glucose is rapidly decomposed, or it may be decomposed during the transfer from blood to saliva. ${ }^{19}$

Campbell MJA observed that the salivary glucose levels were significantly higher in patients with diabetes when compared to nondiabetic healthy individuals. However, no significant correlation was observed between blood glucose and salivary glucose levels. ${ }^{27}$ Similarly, a study conducted by Forbat et al reported no significant correlation between blood and salivary glucose levels. ${ }^{28}$

Nakamoto et al compared the levels of salivary glucose among healthy controls, patients with impaired glucose tolerance test (IGTT) and patients with diabetes. The study revealed no correlation between salivary glucose and plasma glucose levels. ${ }^{19}$

Manjrekar et al compared the levels of salivary glucose among healthy controls, nondiabetic first-degree relatives of patients with type II diabetes, and patients with type II diabetes. Salivary glucose levels did not correlate with plasma glucose levels in all the groups. ${ }^{29}$

In the present study, we did not assess salivary glucose levels as extensive literature review shows there is no correlation between salivary and plasma glucose levels. ${ }^{19}$

Salivary fructosamine is a glycated protein formed by the nonenzymatic reaction between glucose and plasma proteins (mostly albumin). ${ }^{11}$ Since the protein combines with glucose, it is relatively stable against bacteria when compared to salivary glucose. A substantial body of evidence shows that plasma fructosamine is correlated with plasma glucose and HbA1C levels; however, very few studies have been conducted to estimate the levels of salivary fructosamine in diabetic patients. ${ }^{19}$ Hence the present study was contemplated to determine the association between salivary fructosamine and plasma glycated hemoglobin (HbA1C), plasma fasting, and postprandial glucose levels among patients with type II diabetes mellitus and nondiabetic controls. 
Table 1 Distribution based on the demographic detail and knowledge about plasma HbA1C test of the study population

\begin{tabular}{|c|c|c|c|c|c|}
\hline \multirow{2}{*}{$\begin{array}{l}\text { Variables } \\
\text { Age }\end{array}$} & \multicolumn{2}{|c|}{ Group I } & \multicolumn{2}{|c|}{ Group II } & \multirow{2}{*}{$\begin{array}{l}p \text {-Value } \\
\mathbf{0 . 0 8 4 ^ { \# }}\end{array}$} \\
\hline & $\begin{array}{l}N \\
50\end{array}$ & $\begin{array}{l}\text { Mean }( \pm S D) \\
54.38( \pm 9.924)\end{array}$ & $\begin{array}{l}N \\
50\end{array}$ & $\begin{array}{l}\text { Mean }( \pm S D) \\
50.90( \pm 9.990)\end{array}$ & \\
\hline $\begin{array}{l}\text { Sex } \\
\text { Male } \\
\text { Female }\end{array}$ & $\begin{array}{l}N \\
30 \\
20\end{array}$ & $\begin{array}{l}\% \\
60 \\
40\end{array}$ & $\begin{array}{l}N \\
21 \\
29\end{array}$ & $\begin{array}{l}\% \\
42 \\
58\end{array}$ & $0.072^{*}$ \\
\hline $\begin{array}{l}\text { Race } \\
\text { Aryo-Dravidian } \\
\text { Dravidian } \\
\text { Indo-Aryan }\end{array}$ & $\begin{array}{l}14 \\
32 \\
4\end{array}$ & $\begin{array}{l}28 \\
64 \\
8\end{array}$ & $\begin{array}{l}13 \\
36 \\
1\end{array}$ & $\begin{array}{l}26 \\
72 \\
2\end{array}$ & $0.417^{* *}$ \\
\hline $\begin{array}{l}\text { Tobacco Usage } \\
\text { Yes } \\
\text { No }\end{array}$ & $\begin{array}{l}17 \\
33\end{array}$ & $\begin{array}{l}34 \\
66\end{array}$ & $\begin{array}{l}14 \\
36\end{array}$ & $\begin{array}{l}28 \\
72\end{array}$ & $0.517^{*}$ \\
\hline $\begin{array}{l}\text { Alcohol Usage } \\
\text { Yes } \\
\text { No }\end{array}$ & $\begin{array}{l}12 \\
38\end{array}$ & $\begin{array}{l}24 \\
76\end{array}$ & $\begin{array}{l}9 \\
41\end{array}$ & $\begin{array}{l}18 \\
82\end{array}$ & $0.461^{*}$ \\
\hline $\begin{array}{l}\text { Family History } \\
\text { Yes } \\
\text { No }\end{array}$ & $\begin{array}{l}34 \\
16\end{array}$ & $\begin{array}{l}68 \\
32\end{array}$ & $\begin{array}{l}10 \\
40\end{array}$ & $\begin{array}{l}20 \\
80\end{array}$ & $<0.001^{*}$ \\
\hline $\begin{array}{l}\text { Knowledge about Plasma HbA1C Test } \\
\text { Yes } \\
\text { No }\end{array}$ & $\begin{array}{l}32 \\
18\end{array}$ & $\begin{array}{l}64 \\
36\end{array}$ & $\begin{array}{l}24 \\
26\end{array}$ & $\begin{array}{l}48 \\
52\end{array}$ & $0.107^{*}$ \\
\hline
\end{tabular}

* Pearson chi-square test

**Fisher's exact test

"T-test.

Table 2 Distribution based on the mean BMI, plasma fasting and postprandial glucose, plasma glycated hemoglobin, and salivary fructosamine levels of the study population

\begin{tabular}{|c|c|c|c|c|c|c|c|}
\hline \multirow{4}{*}{$\begin{array}{l}\text { Variables } \\
\text { BMI }\end{array}$} & \multicolumn{3}{|l|}{ Group I } & \multicolumn{3}{|l|}{ Group II } & \multirow{4}{*}{$\begin{array}{l}p \text {-Value } \\
<0.001^{*}\end{array}$} \\
\hline & Male & $\begin{array}{l}N \\
30\end{array}$ & $\begin{array}{l}\text { Mean }( \pm S D) \\
24.83( \pm 1.147)\end{array}$ & Male & $\begin{array}{l}N \\
21\end{array}$ & $\begin{array}{l}\text { Mean }( \pm S D) \\
22.71( \pm 1.521)\end{array}$ & \\
\hline & Female & 20 & $24.90( \pm 1.021)$ & Female & 29 & $22.55( \pm 1.804)$ & \\
\hline & $p$-Value & & $0.918^{*}$ & $p$-Value & & $0.787^{*}$ & \\
\hline \multirow[t]{3}{*}{ Plasma Fasting Glucose (mg/dL) } & Male & 30 & $128.20( \pm 46.643)$ & Male & 21 & $84.14( \pm 6.792)$ & \multirow[t]{3}{*}{$<0.001^{*}$} \\
\hline & Female & 20 & $167.60( \pm 68.775)$ & Female & 29 & $84.55( \pm 5.968)$ & \\
\hline & $p$-Value & & 0.014 & $p$-Value & & $0.738^{*}$ & \\
\hline \multirow{3}{*}{$\begin{array}{l}\text { Plasma Postprandial Glucose } \\
\text { (mg/dL) }\end{array}$} & Male & 30 & $182.77( \pm 96.524)$ & Male & 21 & $110.19( \pm 14.713)$ & \multirow[t]{3}{*}{$<0.001^{*}$} \\
\hline & Female & 20 & $259.35( \pm 105.068)$ & Female & 29 & $115.21( \pm 15.486)$ & \\
\hline & $p$-Value & & $0.011^{*}$ & $p$-Value & & $0.288^{*}$ & \\
\hline \multirow{3}{*}{$\begin{array}{l}\text { Plasma HbA1c } \\
(\%)\end{array}$} & Male & 30 & $6.53( \pm 2.097)$ & Male & 21 & $4.90( \pm 0.539)$ & \multirow[t]{3}{*}{$<0.001^{*}$} \\
\hline & Female & 20 & $8.35( \pm 2.700)$ & Female & 29 & $4.66( \pm 0.484)$ & \\
\hline & $p$-Value & & $0.019^{* *}$ & $p$-Value & & $0.111^{* *}$ & \\
\hline \multirow{3}{*}{$\begin{array}{l}\text { Salivary Fructosamine } \\
(\mu \mathrm{mol} / \mathrm{L})\end{array}$} & Male & 30 & $158.80( \pm 97.736)$ & Male & 21 & $80.90( \pm 29.348)$ & \multirow[t]{3}{*}{$<0.001^{* *}$} \\
\hline & Female & 20 & $208.10( \pm 72.480)$ & Female & 29 & $85.07( \pm 25.209)$ & \\
\hline & $p$-Value & & $0.036^{*}$ & $p$-Value & & $0.582^{*}$ & \\
\hline
\end{tabular}

"Mann-Whitney $U$ test.

Gen et al conducted a study among diabetic patients and healthy controls. The study results reported that salivary fructosamine levels were significantly higher in individuals with diabetes $(0.61 \pm 0.19 \mathrm{mmoL} \mathrm{DMF} / \mathrm{L})$ when compared to healthy controls $(0.38 \pm 0.07 \mathrm{mmol} \mathrm{DMF} / \mathrm{L})$. Salivary fructosamine was positively correlated with plasma fasting glucose $(r=0.449)$, plasma fructosamine $(r=0.526)$, and plasma glycated HbA1C $(r=0.411) .{ }^{30}$

Nakamoto et al reported the mean salivary fructosamine levels of the study population as $25.2 \pm 11.6 \mu \mathrm{mol} / \mathrm{g}$ protein. A positive correlation was observed between salivary fructosamine, plasma $\mathrm{HbA} 1 \mathrm{C}$, and plasma blood glucose 
Table 3 Correlation between salivary fructosamine, plasma fasting and postprandial glucose, and plasma glycated hemoglobin levels in the two groups

\begin{tabular}{|l|l|l|l|}
\hline \multicolumn{2}{|l|}{} & Salivary fructosamine (umol/L) & \\
\hline Groups & Variable & r-Value (Spearman's rho) & -Value \\
\hline \multirow{3}{*}{ Group I } & Plasma fasting glucose $(\mathrm{mg} / \mathrm{dL})$ & 0.934 & $<0.001$ \\
\cline { 2 - 4 } & Plasma postprandial glucose $(\mathrm{mg} / \mathrm{dL})$ & 0.910 & $<\mathbf{0 0 1}$ \\
\cline { 2 - 4 } & Plasma HbA1C $(\%)$ & 0.893 & $<\mathbf{0 0 1}$ \\
\hline \multirow{3}{*}{ Group II } & Plasma fasting glucose $(\mathrm{mg} / \mathrm{dL})$ & 0.794 & $<\mathbf{0 0 1}$ \\
\cline { 2 - 4 } & Plasma postprandial glucose $(\mathrm{mg} / \mathrm{dL})$ & 0.530 & $\mathbf{0 . 0 0 1}$ \\
\cline { 2 - 4 } & Plasma HbA1C $(\%)$ & 0.307 & $\mathbf{0 . 0 3 0}$ \\
\hline
\end{tabular}

levels ( $r=0.449$, and $r=0.445$, respectively). No association was observed between salivary fructosamine and plasma fructosamine $(r=0.260)$. This was attributed to the protein compensation that occurs in the oral cavity (by the combination of salivary proteins and glucose); however, such protein compensation was not observed in plasma fructosamine. The study suggests that since no correlation was observed between salivary glucose and plasma glucose, the correlation between salivary fructosamine, plasma glucose, and HbA1C reflects that the salivary fructosamine was produced in the blood or in saliva over a short period of time. ${ }^{19}$

Morenkova reported that there was an increase in salivary fructosamine concentration with an increase in plasma glucose levels. The levels of salivary fructosamine in healthy controls, patients with type I diabetes, and patients with type II diabetes were $3.7 \pm 0.7 \mathrm{mmol} / \mathrm{L}, 6.2 \pm 1.7 \mathrm{mmol} / \mathrm{L}$, and $7.3 \pm 2.0 \mathrm{mmol} / \mathrm{L}$, respectively. ${ }^{31}$

Manjrekar et al, reported that diabetic patients had significantly higher levels of salivary fructosamine (202.1 \pm $103.4 \mathrm{mg} / \mathrm{dL}$ ) when compared to first-degree relatives (130 \pm $71.6 \mathrm{mg} / \mathrm{dL}$ ) and control group (99.8 $\pm 50.1 \mathrm{mg} / \mathrm{dL}$ ). However, no significant correlation was observed between salivary fructosamine and plasma fasting glucose levels in all the groups group I $r$ value $=0.061$, group II $r$ value $=0.171$, group III $r$ value $=-0.078) .{ }^{29}$

In the present study, patients with type II diabetes had significantly higher levels of salivary fructosamine when compared to nondiabetic healthy individuals ( $p$ value $<0.001$ ). The mean levels of salivary fructosamine among group I male and female participants were observed to be 158.80 $( \pm 97.736)$ and $208.10( \pm 72.480) \mu \mathrm{mol} / \mathrm{L}$, respectively. Among group II male and female participants the mean levels of salivary fructosamine were observed to be $80.90( \pm 29.348)$ and $85.07( \pm 25.209) \mu \mathrm{mol} / \mathrm{L}$, respectively. It was also observed that female participants in group I had significantly higher levels of salivary fructosamine when compared to male participants ( $p$-value $=0.036$ ). The higher levels of salivary fructosamine among group I female participants can be attributed to the significantly higher levels of plasma fasting and postprandial glucose levels in them, which in turn leads to increased glycation of plasma proteins.

Participants in group I had significantly higher levels of plasma fasting glucose when compared to group II ( $p$-value < 0.001); also in Group I female participants had significantly higher levels of plasma fasting glucose when compared to male participants $(p$-value $=0.014)$. This may be attributed to the difference in insulin sensitivity, insulin secretion, and hepatic glucose production, among the two genders. ${ }^{32,33}$

A high correlation was observed between salivary fructosamine and plasma fasting glucose levels in group I $(r=0.934)$ and group II $(r=0.794)$ individuals.

Participants in group I had significantly higher levels of plasma postprandial glucose when compared to group II ( $p$ value $<0.001$ ); also in group I female participants had significantly higher levels of plasma postprandial glucose when compared to male participants ( $p$ value $=0.011$ ). This may be attributed to the prolonged gut absorption of glucose in women. ${ }^{33}$

A high correlation was observed between salivary fructosamine and plasma postprandial glucose levels in group I $(r=0.910)$. In group II, a moderate correlation was observed between salivary fructosamine and plasma postprandial glucose levels $(r=0.530)$ individuals.

Participants in group I had significantly higher levels of plasma HbA1C when compared to group II ( $p$ value $<0.001$ ); also in group I female participants had significantly higher levels of plasma HbA1C when compared to male participants ( $p$ value $=0.019)$. Glycemic and metabolic control in women are affected by postprandial glucose levels. ${ }^{33}$ Hence the higher HbA1C levels among female participants may be attributed to the high levels of fasting and postprandial glucose levels, which in turn leads to increased glycation of hemoglobin. ${ }^{33}$

A high correlation was observed between salivary fructosamine and plasma HbA1C levels in group I $(r=0.893)$, and in group II, a moderate correlation was observed $(r=0.307)$.

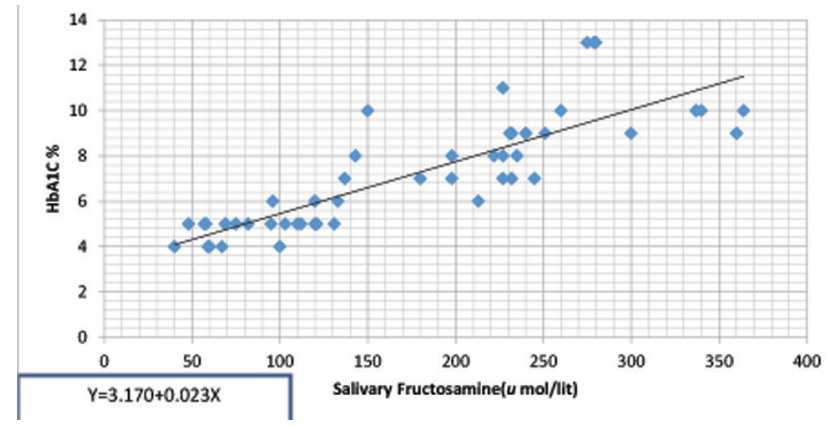

Fig. 1 Correlation between salivary fructosamine and plasma glycated hemoglobin levels in group I individuals. 


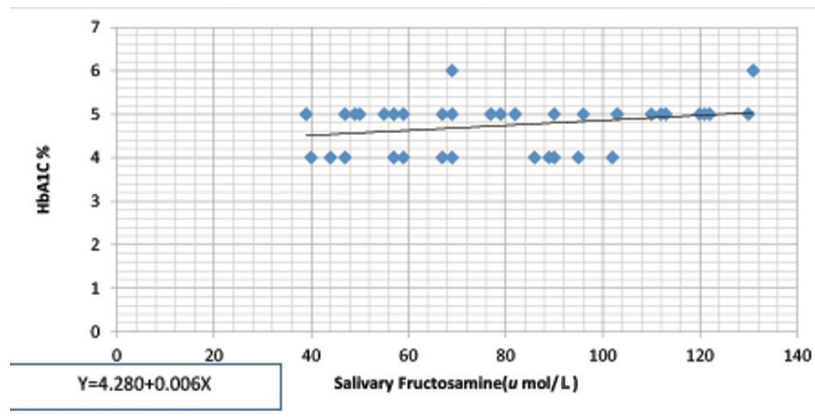

Fig. 2 Correlation between salivary fructosamine and plasma glycated hemoglobin levels in group II individuals.

Saliva collection apart from being simple and noninvasive can be utilized in instances where the collection of blood is not ideal due to the patient's age (especially in pediatric and older adults), attitude toward venous puncture, and hygiene issues in rural areas. ${ }^{34}$ Collection of saliva does not require any special training or equipment. Hence salivary biomarkers can be used in large-scale population assessments and screening programs. ${ }^{34}$

The limitations of the study include the use of convenience sampling technique with cross-sectional design; hence further longitudinal studies are required to validate the use of salivary fructosamine for the diagnosis and monitoring of glycemic control in patients with diabetes.

\section{Conclusion}

The present study throws light on the potential use of salivary fructosamine for the diagnosis and post-treatment monitoring of glycemic control among patients with diabetes. The overall study results showed that salivary fructosamine levels were significantly higher in patients with type II diabetes mellitus when compared to nondiabetic individuals. Also, a positive correlation was observed between salivary fructosamine, plasma fasting, plasma postprandial, and plasma glycated hemoglobin.

\section{Funding \\ None.}

\section{Conflict of Interest}

None declared.

\section{References}

1 International Diabetes Federation. Diabetes Atlas. 6th ed. Belgium: International Diabetes Federation; 2013

2 Yesudian CAK, Grepstad M, Visintin E, Ferrario A. The economic burden of diabetes in India: a review of the literature. Global Health 2014;10(80):80

3 Patel P, Macerollo A. Diabetes mellitus: diagnosis and screening. Am Fam Physician 2010;81(7):863-870

4 Subramani R, Devi U, Shankar U, Rajalakshmi R, Seshadhri, Karthick. Prevalence of undiagnosed type 2 diabetes and its associated risk factors in rural population of Tamil Nadu. World J Med Sci 2014;11(2):222-227

5 Maynard JD, Rohrscheib M, Way JF, Nguyen CM, Ediger MN. Noninvasive type 2 diabetes screening: superior sensitivity to fasting plasma glucose and A1C. Diabetes Care 2007;30(5): 1120-1124

6 World Health Organization. Screening for Type 2 Diabetes. Report of a World Health Organization and International Diabetes Federation Meeting. Geneva, Switzerland: World Health Organization; 2003 http://www.who.int/diabetes/publications/en/screening_mnc03.pdf. Accessed on 01/02/2016

7 American Diabetes Association. Standards of medical care in diabetes 2014. Diabetes Care 2014;37(1):14-80

8 Schwartz JG, Phillips WT, Aghebat-Khairy B. Revision of the oral glucose tolerance test: a pilot study. Clin Chem 1990;36(1):125-128

9 Youssef D, El Abbassi A, Jordan RM, Peiris AN. Fructosaminean underutilized tool in diabetes management: case report and literature review. Tenn Med 2008;101(11):31-33

10 Singh R, Barden A, Mori T, Beilin L. Advanced glycation end-products: a review. Diabetologia 2001;44(2):129-146

11 Armbruster DA. Fructosamine: structure, analysis, and clinical usefulness. Clin Chem 1987;33(12):2153-2163

12 Bonora E, Tuomilehto J. The pros and cons of diagnosing diabetes with A1C. Diabetes Care 2011;34(2, Suppl 2): S184-S190

13 Mula-Abed WA, Al-Naemi AH. Performance indicators and validity of serum fructosamine assay as a diagnostic test in a screening program for diabetes mellitus. Saudi Med J 2003;24(5):477-484

14 Sahibzada HA, Khurshid Z, Khan RS, et al. Salivary IL-8, IL-6 and TNF- $\alpha$ as Potential Diagnostic Biomarkers for Oral Cancer. Diagnostics (Basel) 2017;7(2):2-10

15 Abdul Rehman S, Khurshid Z, Hussain Niazi F, et al. Role of Salivary Biomarkers in Detection of Cardiovascular Diseases (CVD) Proteomes 2017;5(3):5-6

16 Khurshid Sultan Z, Khan E. Future of oral proteomics. J Oral Res 2018;7(2):42-43

17 Neqoro H, Morley J, Rosenthal MJ. Utility of serum fructosamine as a measure of glycaemia in young and old diabetic and non-diabetic subjects. Am J Med 1998;85(3):360-364

18 Streckfus C, Bigler L. The use of soluble, salivary c-erbB-2 for the detection and post-operative follow-up of breast cancer in women: the results of a five-year translational research study. Adv Dent Res 2005;18(1):17-24

19 Nakamoto I, Morimoto K, Takeshita T, Toda M. Correlation between saliva glycated and blood glycated proteins. Environ Health Prev Med 2003;8(3):95-99

20 Khurshid Z, Zohaib S, Najeeb S, Zafar MS, Slowey PD, Almas K. Human saliva collection devices for proteomics: an update. Int J Mol Sci 2016;17(6):7-10

21 Gröschl M, Rauh M. Influence of commercial collection devices for saliva on the reliability of salivary steroids analysis. Steroids 2006;71(13-14):1097-1100

22 Sannam Khan R, Khurshid Z, Akhbar S, Faraz Moin S. Advances of salivary proteomics in oral squamous cell carcinoma (OSCC) detection: an update. Proteomes 2016;4(4):41

23 Khurshid Z, Moin SF, Khan RS, Agwan MAS, Alwadaani AH, Zafar MS. Human salivary protein extraction from RNAPro SAL, Pure SAL, and passive drooling method. Eur J Dent 2017;11(3):385-389

24 Radha V, Mohan V. Genetic predisposition to type 2 diabetes among Asian Indians. Indian J Med Res 2007;125(3):259-274

25 Mohan V. Why are Indians more prone to diabetes? J Assoc Physicians India 2004;52(1):468-474

26 Nowotny K, Jung T, Höhn A, Weber D, Grune T. Advanced glycation end products and oxidative stress in type 2 diabetes mellitus. Biomolecules 2015;5(1):194-222

27 Campbell MJA. Glucose in the saliva of the non-diabetic and the diabetic patient. Arch Oral Biol 1965;10(1):197-205 
28 Forbat LN, Collins RE, Maskell GK, Sönksen PH. Glucose concentrations in parotid fluid and venous blood of patients attending a diabetic clinic. J R Soc Med 1981;74(10):725-728

29 Manjrekar PA, Hedge A, Shrilaxmi D, Souza F, Kaveeshwar V, Jose A. Fructosamine in non-diabetic first degree relatives of type 2 diabetes patients: risk assessor. J Clin Diagn Res 2012;6(5):770-773

30 Gen Z-R, et al. Measurements of salivary dructosamine and its clinical application. Shangai Medical Journal 1990;11(1):628-631

31 Morenkova SA. [Comparative analysis of dependence of saliva sorbitol and fructosamine levels on blood glucose level in patients with diabetes]. Biomed Khim 2004;50(6):612-614
32 Kautzky-Willer A, Brazzale AR, Moro E, et al. Influence of increasing BMI on insulin sensitivity and secretion in normotolerant men and women of a wide age span. Obesity (Silver Spring) 2012;20(10):1966-1973

33 Kautzky-Willer A, Kosi L, Lin J, Mihaljevic R. Gender-based differences in glycaemic control and hypoglycaemia prevalence in patients with type 2 diabetes: results from patient-level pooled data of six randomized controlled trials. Diabetes Obes Metab 2015;17(6):533-540

34 Rao PV, Laurie A, Bean ES, Roberts CT Jr, Nagalla SR. Salivary protein glycosylation as a noninvasive biomarker for assessment of glycemia. J Diabetes Sci Technol 2015;9(1):97-104

Erratum: The article has been corrected as per erratum published on August 12, 2019. DOI of the erratum is 10.1055/s-0039-1695080. Author Sadhana Kandavel's affiliation was appearing incorrectly. Author's correct affiliation appears as in the article. 